\title{
HISTOIRE SOCIALE
}

Des Ordres à Rome. Sous la dir. de Claude Nicolet. Paris, Publications de la Sorbonne, 1984. $16 \times 24,280$ p. («Publications de la Sorbonne. Histoire ancienne et médiévale ", 13).

Ce livre, publié sous la direction de Claude Nicolet et dont il a rédigé deux chapitres, réunit les travaux de plusieurs auteurs. Certains de ces auteurs sont ses élèves (Marianne Bonnefond, Ségolène Demougin, John Scheid, Catherine Virlouvet), d'autres des collègues scientifiquement proches de lui (André Chastagnol, Benjamin Cohen); tous s'intéressent ici à la définition, au recrutement et au fonctionnement des ordres, qui caractérisaient la hiérarchie civile et administrative de Rome à la fin de la République et sous le Haut Empire.

Dans les années 1960 , la notion d'ordres a pris une importance historiographique notable, en particulier à l'initiative de Roland Mousnier, qui opposait les sociétés d'ordres aux sociétés de classes, et qui, non sans raison, insistait, contre un marxisme très économiciste, sur l'importance des ordres dans les sociétés préindustrielles. Les recherches de Nicolet sur l'ordre équestre (L'Ordre équestre à l'époque républicaine, 2 vols, Paris, de Boccard, 1964 et 1974) révélaient des préoccupations proches de celles de Mousnier, dont elles se réclamaient. Elles ont contribué à persuader les Antiquisants de l'importance sociale de ces groupements, qu'on rencontre à la fois dans l'Antiquité (mais non pas dans toute l'Antiquité) et dans la France d'Ancien Régime.

Vingt ans après, le débat n'est plus entre ordres et classes : ceux qui s'inspirent du marxisme (en histoire ancienne, ils sont plutôt plus nombreux qu'il y a un quart de siècle) sont en général conscients de la complexité du problème ; et les auteurs de ce livre ne cherchent nullement, pour mieux démontrer l'éminence des ordres, à nier l'éventuelle existence de classes sociales. Les questions se sont déplacées, et elles concernent plutôt la signification respective des divers ordres et le fonctionnement de chacun d'entre eux, le rôle que chacun d'entre eux remplit de fait dans l'ensemble du mécanisme social.

Benjamin Cohen a recensé vingt-sept ou vingt-huit ordres ou groupes d'ordres - appelés ordines en latin. Pourtant, comme le souligne Nicolet dans son introduction, " tous les ordines ne sont pas vraiment des ordines ". Car en plus de ces vingt-sept ou vingt-huit, d'autres apparaissent fugitivement, mais leur existence paraît contestable aux yeux des Latins eux-mêmes - qui d'ailleurs n'hésitaient pas à employer le mot ordines pour désigner ce que nous appelons, de façon vague, des groupes sociaux. À l'intérieur même de ceux qu'a recensés Cohen, la diversité est notable.

Tout ordre a rapport à l'État; c'est un groupe limité ; l'État tient à jour la liste de ses membres et, d'une manière ou d'une autre, il est maître de son recrutement. Mais alors que certains ordres, par exemple ceux des « appariteurs " (c'est-à-dire des administrateurs adjoints aux magistrats), qu'étudie 
Cohen dans le présent livre, sont liés à des fonctions précises en l'absence desquelles ils perdraient toute signification, l'ordre des chevaliers, sous la République, constitue une espèce de noblesse, qui n'avait plus d'utilité immédiatement appréciable, mais n'en restait pas moins, avec le Sénat, un des deux grands piliers de l'équilibre social et politique. Les auteurs latins, quoiqu'ils connussent la pluralité des ordres de leur société, tendaient parfois à n'admettre que l'existence des deux plus grands, le Sénat et l'ordre équestre, et parlaient, pour les désigner ensemble, d'uterque ordo, l'un et l'autre des deux ordres.

Le Sénat, pour sa part, est toujours entièrement lié à la vie politique et au fonctionnement de l'Etat. C'est ce qu'illustrent avec brio les deux contributions de Marianne Bonnefond et Catherine Virlouvet. Mais cette fonction constante et prestigieuse n'empêche pas qu'il se soit fortement transformé, évoluant de plus en plus, de la fin de la République au début de l'Empire, vers la constitution d'un groupe plus héréditaire, plus pesant, qui comprenait aussi les épouses et les familles des Sénateurs - ce qui n'était auparavant pas le cas. Les deux chapitres d'André Chastagnol éclairent admirablement cette évolution, dont ils fournissent une chronologie précise.

Lié à l'État, l'ordre forme un groupe de nature juridique. Mais il est en général le fait des milieux privilégiés, et, surtout sous l'Empire, lorsque les oligarchies municipales forment des ordines decurionum, tout membre des milieux privilégiés tend à faire partie d'un ou de plusieurs ordines. À la fin de la République et sous l'Empire, les ordres constituent donc l'armature sociale, administrative et politique de la société romaine, l'institution qui lui permet aussi de recruter ses élites. Mais il n'en a pas toujours été ainsi. Et ce rôle central des ordres n'empêche pas un certain nombre de particularités surprenantes. John Scheid montre par exemple que le recrutement des prêtres (qui étaient pourtant des " laïcs ", la plupart du temps des magistrats romains ou des notables des cités) n'a rien à voir avec l'appartenance à un ordre. De son côté, Ségolène Demougin analyse l'emploi et la signification d'un signe de prestige social, les anneaux d'or, traditionnellement liès à l'ordre équestre. Ses remarquables analyses, qui parcourent en tout sens la forêt des significations de cet emblème, au gré des informations fragmentaires fournies par les inscriptions et textes antiques, montrent que si les ordres se caractérisaient par des emblèmes spécifiques (par des vêtements, par exemple, ou par des insignes), les anneaux d'or n'étaient pas portés par tous les chevaliers et que certains non-chevaliers recevaient de l'Empereur l'autorisation légale de les porter. Ce qui, du reste, n'empêchait pas d'autres non-chevaliers de les porter illégalement, et l'Etat de tolérer cette illégalité.

Société d'ordres? Société de classes ? Société de castes ? Ces catégories stimulantes peuvent, si l'on n'y prend garde, se muer en mots d'ordre abstraits, aux dépens du renouvellement des questions historiques. Ce livre, à la fois érudit et très intéressant à lire, a le grand mérite de montrer tout ce qu'implique en pratique la notion d'ordre, dans le fonctionnement et la reproduction de l'organisation sociale et politique, tous les problèmes qu'elle pose et tous ceux qu'elle aide à résoudre. On ne saurait trop en conseiller la lecture, non seule- 
ment aux amateurs du monde romain, mais à ceux de l'ancienne France : l'un des ouvrages les plus utiles à la connaissance de cette institution romaine n'est-il pas en effet, comme l'écrit Nicolet, le Traité des ordres et simples dignités de Charles Loyseau, publié en 1610 ?

Jean ANDREAU.

L'Âge d'or du mécénat : 1598-1661. Colloque international, Paris, mars 1983, par Roland Mousnier et Jean Mesnard. Paris, C.N.R.S., 1985. $16 \times 24$, $440 \mathrm{p}$.

La publication des actes de ce grand colloque, consacré à l'Âge d'or du mécénat (1598-1661), vient parachever l'œuvre de ces trois jours pendant lesquels se sont succédé de brillantes communications. Ce colloque a eu avant tout le mérite de faire le point sur un phénomène de l'histoire sociale et esthétique qui, pour être connu, n'en restait pas moins difficile à saisir.

Non, bien sûr, que la réponse que constituent ces actes doive être tenue pour définitive, mais il faut toutefois reconnaître à ce colloque l'avantage de présenter clairement l'enjeu culturel et politique qu'a constitué le mécénat pour cette période. Comme le constatait d'entrée de jeu Marc Fumaroli, le mécénat est une émanation typique de la société d'Ancien Régime. Car si l'on accepte toujours d'envisager celle-ci comme une société d'ordres, on s'aperçoit, en effet, que la pratique du mécénat s'épanouit pleinement en un temps où la relative stabilité des ordres et des valeurs de la société n'a pas encore cédé le pas au règne triomphant de l'utilitarisme et de la vitesse.

Si le mécénat doit assurément être envisagé dans la perspective d'une dépendance économique entre l'artiste, le savant, l'écrivain et le protecteur, on ne peut cependant pas réduire le mécénat à cet aspect purement mercantile. Car il y a, en outre, une connivence d'esprit et de goût entre le patron et son protégé, et cela parce que les Grands perdent progressivement leur pouvoir politique, judiciaire et militaire au profit d'un absolutisme toujours plus marqué du pouvoir monarchique. Les Grands passent ainsi, selon l'heureuse expression du préfacier, d'une "magistrature civile de la norme " à une "magistrature du goût ". Si le mécénat officiel et royal existe avant tout sous l'aspect du negotium, il peut encore coexister avec celui des Grands en recouvrant aussi la notion d'otium, car le principe des relations qui unissent mécènes et protégés ne se trouve pas modifié. L'urbanité marque profondément les liens de " domesticité " que crée le mécénat. Il est un art de vivre dans la mesure où les principes esthétiques définis par les Grands, et appliqués par les artistes, régissent tout l'ordonnancement de ce grand " thêâtre du monde ".

La première partie du colloque a donc été consacrée aux différents types de mécènes que cette période a connus. Ainsi Hélène Himelfarb nous invite-t-elle à suivre les premiers pas de l'apprentissage du mécénat royal du jeune Louis XIII. Elle nous permet de suivre, grâce au journal d'Héroard, la façon dont, en 1608 , les premières tendances pouvaient déjà expliquer les réalisa- 
tions à venir, et ce, par une habile mise en perspective de celles-ci par rapport aux jeux et aux leçons du petit roi. Tandis que E. Caldicott nous montre comment Monsieur a su, malgré les tribulations de sa vie, satisfaire par le mécénat une curiosité d'esprit très réelle, aidée de surcroît par un goût très sûr. C'est, nous explique-t-on, ce qui a permis à Gaston d'Orléans d'être comme le protecteur d'une expression bien française dans l'art et la littérature, contre un engouement toujours plus prononcé pour tout ce qui venait d'Italie.

Sans quitter le mécénat officiel, mais à côté du roi ou de sa famille, on observe que les cardinaux ministres se sont particulièrement illustrés. W.D. Howarth s'intéresse aux rapports qui ont existé entre Richelieu et Corneille. Il rappelle à ce sujet que le grand Corneille, tout indépendant d'esprit qu'il se soit voulu, a bien fini par manifester dans son théâtre l'idéal politique et moral cher au cardinal, sans pour autant se faire le chantre de Son Éminence. Alors que Madeleine Laurain-Portemer, en soulignant l'importance de l'empreinte romaine sur l'esprit de Mazarin, distingue dans son mécénat trois aspects qui caractérisent l'action du cardinal ministre dans ce domaine : "L'impulsion qui stimule, la protection qui facilite, la fondation qui perpétue. "

Moins directement politique, et pourtant encore très officiel, le mécénat du chancelier Séguier a été très marquant en dépit du manque d'envergure politique du personnage. Car, comme le note Yannick Nexon, outre la longévité remarquable de Séguier à cette charge (1635-1672), le chancelier est un personnage central de l'État. Non seulement c'est le premier personnage de la hiérarchie administrative, qui, à ce titre, représente le roi, mais encore dispose-t-il des revenus considérables de la trésorerie du sceau. Il contrôle, en outre, la censure de la "librairie ". Autant d'éléments qui font du chancelier un point de mire pour toute sorte de sollicitations.

À côté des personnages officiels existe le patronage des Grands, tel celui des Guise auquel s'intéresse J.-P. Babelon, ou celui du plus étranger de ces " princes étrangers " qu'est Lord Arundel, dont le brillant portrait nous est dressé par David Howarth. Dans ce dernier cas, il est intéressant de voir qu'en Angleterre, on peut à la fois être un grand seigneur amateur d'art, au goût averti comme peut l'être celui d'un Howard, mais que l'on peut concurremment avoir le même goût pour l'érudition qu'un « scholar ", sans pour autant estimer que c'est ravaler sa dignité aristocratique.

La seconde partie du colloque s'intéresse à la condition des protégés des mécènes, ainsi qu'aux rapports que clients et patrons entretiennent. Wolfgang Leiner démontre pertinemment que ce n'est pas en fonction de la valeur esthétique de l'œuvre, mais plutôt en fonction de l'adéquation de l'œuvre aux valeurs de la noblesse que l'artiste pourra espérer une pension substantielle. Il lui faudra donc prouver l'honneur et l'utilité de la plume, qui, à défaut d'être l'égale de l'épée, n'en doit pas pour autant souffrir du hautain mépris aristocratique avec lequel on la considère d'ordinaire. Alain Merot, quant à lui, nous livre une intéressante étude sur les commandes des paroisses parisiennes dans la première moitié du XvII siècle. Il nous apprend ainsi que dans chaque paroisse qui, nous précise-t-il, est une "société en miniature ", ce n'est pas, 
comme on l'a cru exagérément, le clergé qui s'est chargé de passer les commandes d'œuvres d'art pour les églises. Dans la plupart des cas, comme l'attestent les archives, ce furent les représentants des conseils de fabrique de la paroisse, les marguilliers, qui se chargèrent de cela. Il s'agit donc ici d'une forme de mécénat collectif qui a été très répandu et très actif. On pourrait $a$ priori être tenté d'apparenter l'action de l'Académie française à ce type de mécénat collectif. Or Claudette Dethez-Sarlet nous indique qu'il n'en est rien, et que, au contraire, la création de l'Académie française marque une rupture nette. Elle n'est plus une institution de mécénat, à la différence de ce que pouvait être encore l'Académie de Charles IX et de Henri III. Elle n'est plus une " instance de production artistique ", son statut même délimite le champ de son activité intellectuelle. Le strict domaine linguistique et littéraire lui est réservé, et cela en vue de ne produire que dictionnaires et grammaires.

Appartenant au fond à ce second ensemble de communications, la belle étude de Jean Mesnard s'intéresse au mécénat scientifique avant l'Académie des sciences (1666). Trois conditions sont absolument requises pour le savant de l'époque : le loisir, l'indépendance et l'argent. Le savant doit en somme être d'abord son propre mécène. C'est pourquoi il appartient souvent au clergé ou plus encore à la noblesse de robe. Si le savant a un protecteur, cela ne vient que d'une manière complémentaire, il est alors le " domestique " d'un Grand, et ce dernier tire gloire ou avantage de sa protection. Mais peut-être plus intéressante encore est la constatation que le mécénat est souvent interne au milieu savant ; c'est le cas, par exemple, de l'hôtel de Thou à Paris. Toutefois, conclut Jean Mesnard, la science n'a pas attiré le mécénat au même degré que l'activité artistique ou littéraire.

Avec la communication d'Hubert Carrier se trouve posée la question du jugement que les contemporains ont porté sur les mécènes, et en l'occurrence sur le cardinal Mazarin. Cette savante communication vient nuancer en contrepoint celle de $\mathbf{M}^{\text {me }}$ Laurain-Portemer. Car s'il est incontestable qu'il y a eu de la part du ministre une action en faveur des lettres et des arts, il faut constater qu'elle a beaucoup bénéficié aux Italiens, comme au reste on s'est plu alors à le dénoncer. Il est bon, d'autre part, de -souligner que la guerre étrangère a suffisamment grevé les finances de l'État pour que les gestes faits par le cardinal ministre pussent aisément compenser le fastueux et généreux souvenir que l'ombre de Richelieu faisait encore planer.

Les intervenants regroupés pour la troisième partie de ce colloque se sont penchés sur les thèmes et les œuvres qui ont animé cet Âge d'or du mécénat. Bernard Beugnot commence cette série de communications en se demandant si, à cette époque, il existe un mythe de Mecenas. Il observe que si la figure historique de Mécène est bien présente pour les esprits du temps, il s'agit plutôt d'une « légende de Mécène qui assure les fondements du mécénat ». Car il y a plus une idéalisation du personnage historique qu'une métamorphose totale du modèle initial. Le souvenir grandi de Mecenas incarne la regénération d'une société qui retrouve un équilibre après de longues guerres civiles grâce à l'action civilisatrice des belles lettres.

Après l'étude de thèmes, tel celui d' "Europe " présenté comme une allégo- 
rie théâtrale de propagande politique, ou encore comme celui de l'Aminta du Tasse, sont envisagés les divers domaines artistiques dans lesquels un mécénat s'est exercé. Cela va de l'oraison funèbre des rois, aux velins, en passant par les arbres fruitiers ou bien la tapisserie, ou mieux encore la musique, avec ce mécène toujours présent que fut Gaston d'Orléans.

Il nous est évidemment impossible d'évoquer l'intérêt et l'originalité de chaque communication, qui, chacune dans sa spécialité, apporte une nuance précieuse à l'intelligence que l'on peut avoir de ce phénomène si typique de la société du $\mathrm{xvII}^{e}$ siècle. L'étude fouillée du mécénat met bien à jour cet aspect du clientélisme qui ne pouvait prendre une telle ampleur que dans une société fortement hiérarchisée, dont la verticalité des liens assurait à la fois la prédominance du pouvoir royal dans le cadre du mécénat officiel des Grands. Mais le mécénat de cet Âge d'or apparu au gré des communications révèle, en outre, un art de vivre, une urbanité des mœurs qui ne pouvait être que parce que le mécène est surtout un homme de goût. Il cherche à se divertir mais aussi à s'instruire. Aussi peut-il apprécier par lui-même la mesure de la protection qui est nécessaire à l'artiste. Il est comme un catalyseur qui stimule et encourage l'artiste, l'écrivain ou le savant par l'importance des subsides qu'il leur accorde. Au fond, comme le dit Jean Mesnard, le mécénat est «l'hommage que le pouvoir rend à la culture ".

Philippe BONOLAS.

François BillacoIs, Le Duel dans la société française des XVIe-XVII siècles. Essai de psychosociologie historique. Paris, E.H.E.S.S., 1986. $16 \times 24,540$ p., bibliogr., index.

Un phénomène social total : le duel. Un historien qui a le goût de bien écrire et qui aime " parler en parabole " en faisant son métier. Le résultat de cette rencontre est un livre épais mais maniable, original mais prudent, bardé de références érudites et de notes infrapaginales tout en restant facile et agréable à lire.

Le plan est simple, comme une vie d'homme : commencement, apogée et déclin précèdent une quatrième partie thématique, sorte de méditation sur le contenu et les destinées de ce corps symbolique que fut le duel dans la société française d'Ancien Régime.

Machiavel et ses contemporains auraient sans doute accepté aisément de reconnaître que la furie française s'incarnait au mieux dans le duel. Au $\mathrm{XV}^{\mathrm{e}}$ siècle, ce dernier est, en effet, une tradition nobiliaìre française, mais il ne produit un code complexe, technique et éthique, qu'au contact des Italiens. Et François Billacois n'a aucun mal à nous convaincre que le duel devient spécifiquement un phénomène français au temps de la Renaissance, comme le montre par exemple le célèbre combat de Jarnac contre La Châtaigneraye en 1547.

L'apogée se situe à la fin du Xvie et au début du Xvil ${ }^{e}$ siècle. Les formes de la rencontre se précisent alors, des seconds étant désormais chargés de s'égorger 
à l'ombre des acteurs principaux. Les oppositions au phénomène croissent brusquement et fortement : l'Église et l'État, les confesseurs et les juges tentent activement de réduire ces occasions où l'homme "faict acte de beste brutte ". Chose difficile, s'il en est, car le duel appartient intensément et profondément à la culture et au style de vie des nobles. Pour les bourgeois, dont les attitudes sont diverses, il a aussi un immense prestige, même pour ceux qui s'affirment et veulent rester bourgeois, tant il est vrai que la société est dominée par des idéaux aristocratiques. L'exécution du comte de Bouteville, coupable d'avoir transgressé les édits contre le duel, en 1627, témoigne de l'existence de ces fortes contradictions.

La fin du duel vient doucement à partir de 1650. Louis XIV est habituellement considéré comme celui qui en a sonné le glas, alors que son action n'a pas été plus efficace que celle de ses prédécesseurs et qu'il n'a peut-être même pas vraiment eu envie de se montrer intransigeant. Tout au plus s'est-il approprié le bénéfice d'une profonde déperdition de substance du duel en son temps. Depuis lors, la fin du phénomène n'a jamais cessé de s'annoncer, sans encore survenir : Gaston Defferre ne défraya-t-il pas la chronique le 22 avril 1967, à la suite de sa victoire, par deux touches à zéro, sur le député Ribière?

À l'évidence, le duel n'eut jamais de graves conséquences démographiques. L'auteur fait justice des exagérations polémiques à ce sujet. Les poursuites n'aboutirent pas non plus à des hécatombes, même sous Louis XIII et Richelieu. L'importance du duel, en fait, est d'une autre nature, que tente de cerner la quatrième partie du livre. L'épée et le sang engagent, en effet, un dialogue qui n'a pas besoin des mots pour exprimer l'appartenance à une même " tribu ", selon l'expression de l'auteur. On lira avec intérêt les développements sur ces thèmes sensibles. Avec quelques réserves cependant, car l'épée n'est nullement un apanage des nobles sous Henri IV et Louis XIII, comme François Billacois le sait sans toujours en tenir compte. L'analyse mériterait de prendre en considération la fascination qu'elle exerce sur les jeunes paysans de la même époque, qui la portent souvent et qui se défient autant que les nobles eux-mêmes : cette dimension du duel n'apparaît pas dans l'ouvrage. Par ailleurs, opposer l'épée au bâton (tableau p. 332) est un peu artificiel, quand l'on sait que la première était souvent nommée «baston " ou «baston esmolu » dans les provinces francophones des Pays-Bas de Philippe II. Quant au sang du combat, il me paraît, en effet, être au centre d'une extraordinaire symbolique, nullement propre aux aristocrates d'ailleurs; d'où une certaine frustration à la lecture des pages trop brèves et trop imprécises qui lui sont consacrées.

Suivent trois thèmes éthiques : honneur, justice, générosité et fraternité, puis trois thèmes théologico-politiques : la guerre, la croisade, le page et le roi. Ils décrivent la fonction initiatique du duel, rite de passage de la jeunesse à l'âge mûr, symbole de la confrontation entre le fils et le père, défi porté à la mort tout autant qu'au roi, dont le meurtre impossible est pourtant réalisé par personne interposée, lorsque tombe l'adversaire, sur ce théâtre où s'expriment les idéaux de l'aristocratie. Résumer ces notions est déjà les déformer : mieux vaut méditer les pages convaincantes que leur consacre François Billacois. 
En refermant le livre, je m'avoue séduit par la démonstration. D'un événement somme toute marginal, dans tous les sens du mot, l'historien fait habituellement le point de départ de l'analyse d'une minorité résistante, puis du corps social - l'aristocratie, mais aussi la Cour et la ville - dans lequel elle est immergée. Cependant, une certaine insatisfaction affleure à son tour. Le temps des duels est aussi en France l'âge baroque, celui des révoltes populaires, l'ère de l'invasion mystique, l'époque d'une frénésie de persécution des sorciers. Nul ne le sait mieux que l'auteur, qui avoue en conclusion que des rapprochements seraient à faire (p. 398). Que ne les a-t-il pas au moins esquissés?

Brillant, solide, son livre vise à épuiser un sujet. Il est de ce fait un peu trop " fermé " sur le monde du duel, qu'il aurait fallu replacer dans le cadre de la " civilisation des mœurs " étudiée par Norbert Élias. La brutalité des nobles désamorcée par un tel processus, où le duel prend sa place comme instrument 'd'acceptation de l'ordre monarchique moderne, méritait plus qu'une allusion (p. 397). Par ailleurs, si les duellistes veulent tuer le père-roi (p. 387), quelle est la figure culturelle de ce dernier en ce temps? Car il est douteux qu'il ressemble au modèle paternel du $x^{e}$ siècle à l'œuvre dans les ouvrages de Freud et de ses successeurs.

L'interprétation politique finale du duel comme "bricolage culturel " et adaptation de la noblesse à une situation nouvelle, attitude non pas crispée et réactionnaire mais inventive d'une stratégie symbolique neuve pour signifier un contre-pouvoir au sein du système politique existant (p. 391), me paraît à la fois judicieuse et féconde. Je n'en regrette que plus de la voir s'arrêter court, sans prospecter ce champ de l'adaptation aristocratique, par échanges, conflits ou créations, à l'émergence de l'absolutisme. La bibliographie, antérieure sauf exception au milieu des années 1970 , explique quelque peu cela. Les exigences académiques sont d'ailleurs telles que l'analyse d'un semblable sujet aurait exigé des années de labeur supplémentaires : on peut comprendre qu'un auteur limite de ce fait ses ambitions en refermant un dossier qu'il a parfaitement analysé.

Qu'il le veuille ou non, d'ailleurs, François Billacois ouvre la piste d'une enquête immense, passionnante et difficile: le duel, forme de «bricolage culturel " propre à l'aristocratie, est le révélateur d'une mutation profonde et définitive des rapports entre les pouvoirs et les sujets, fussent-ils nobles. Ces derniers refusent au fond de nouvelles formes d'autorité, que la justice leur impose en partie, lorsque le roi ne défait pas d'une main ce qu'il a institué de l'autre. Comme les procès de sorcellerie, la répression des révoltes populaires ou l'exercice dè la justice criminelle en général, les poursuites contre les duels transcrivent la cristallisation symbolique qui s'opère dans les divers groupes de la société face à un exercice du pouvoir fondamentalement différent de celui du passé. L'homme moderne est en train de naître, douloureusement et laborieusement, dans les contradictions de toutes natures. En ce sens, la répression du duel fait partie des multiples procédures de formation du consentement, ou en d'autres termes de conditionnement, des sujets, comme le duel lui-même en exprime le refus. Il reste à explorer; à comparer l'évolution 
des « forces d'ailleurs » qui motivent ces phénomènes, pour bâtir une anthropologie culturelle et politique de la France moderne. François Billacois en a posé l'une dès premières pierres, bien qu'il n'ait pas voulu dessiner le plan de la construction.

Robert MUCHEMBLED.

L'Absolutisme éclairé. Vol. pub1. par B. KöPeczI, A. Soboul, É. H. BalÁzs, D. KosÁry. Budapest/Paris, Akadémiai Kiadó/C.N.R.S., 1985. $17 \times 25$, 364 p., bibliogr. (« Colloques de Mátrafüred»).

Sous l'intitulé général "Études sur les Lumières", la Société hongroise d'étude $d u x^{\prime}{ }^{\circ}{ }^{e}$ siècle organise tous les trois ans un colloque international à Mátrafüred. En 1975 et 1978, les troisième et quatrième rencontres furent consacrées à l' " absolutisme éclairé ». Ce sont les rapports et contributions qui se trouvent réunis dans ce volume. On peut déplorer le caractère tardif de cette publication collective. L' "absolutisme éclairé " ayant occupé une séance du Colloque organisé à Paris en 1978 (bicentenaire de la mort de Voltaire et Rousseau), certains textes figurent, en effet, dans un numéro spécial des Annales historiques de la Révolution française (1979): ainsi le rapport d'A. Soboul "Sur la fonction historique de l'absolutisme éclairé ", ou l'original «cas danois" analysé par K. Tónnesson. D'autres contributions, en revanche, ont été largement remaniées et amplifiées, telle l'étude des pays italiens par F. Diaz ou celle de la Hongrie par É. Balázs. Retenons donc surtout ce qui peut être moins connu des dix-huitiémistes français et posons d'emblée le problème de l'intitulé même du livre : "despotisme " ou " absolutisme " éclairé ?

“ L'absolutisme existe-t-il ? " interrogeait Michèle Fogel en introduction à sa thèse, Le Roi et les armes. Recherches sur l'absolutisme et ses moyens symboliques de domination ... ${ }^{1}$. Et M. Fogel rappelait les débats de l'historiographie depuis la contribution essentielle de $\mathrm{F}$. Hartung et $\mathrm{R}$. Mousnier au $\mathrm{X}^{\mathrm{e}}$ Congrès international des Sciences historiques de Rome, en 1955. On sait que P. Goubert refuse le terme d' "absolutisme" ("inutile et creux") ${ }^{2}$, tandis que R. Mandrou emploie des guillemets (Europe «absolutiste», monarchie " absolue ") ${ }^{3}$ et que D. Richet souligne les " ambiguités de l'absolutisme " 4 . II est donc un peu regrettable que le comité de rédaction ait abandonné l'idée de publier dans ce volume les travaux relatifs au problème de l'État (« Avant-propos » de Béla Köpeczi, p. 8) : le politique n'est ainsi abordé que par le biais de l'idéologie (B. Köpeczi, p. 101-118) ou des exemples régionaux. Pour l'essen-

\footnotetext{
1. Thèse de 3 e cycle, Université de Paris I, 1979.

2. Pierre Goubert, L'Ancien Régime. t. II : Les Pouvoirs, Paris, A. Colin, 1973, p. 12.

3. Robert MANDROU, L'Europe " absolutiste ", Paris, Fayard, 1977.

4. Denis Richet, La France moderne: l'esprit des institutions, Paris, Flammarion, 1973 , p. 37. Ce livre et celui de P. Goubert ne figurent pas dans la bibliographie qui clôt le volume dont nous rendons compte.
} 
tiel toutefois, hormis la contribution de Jean Meyer consacrée à " La noblesse » (p. 51-57) et en dépit de divergences théoriques, les auteurs semblent faire leur la définition de l'absolutisme proposée par Perry Anderson : « un nouvel aménagement, un renforcement de l'appareil de la domination féodale $"{ }^{6}$. Absolutisme, donc, au sens où le système répond dans son principe à la maxime des juristes "Princeps legibus solutus est " et à condition aussi d'entendre loi dans l'acception du droit naturel, en particulier de John Locke : ce qui est " indispensable " à une loi " pour qu'elle soit une loi ", c'est "le consentement de la société " (Deuxième Traité du Gouvernement civil, chap. XI, § 134).

Le volume est organisé de façon très " classique ": les vingt-deux contributions sont distribuées en deux grandes rubriques, "Problèmes de base " (des " structures " aux "superstructures ") et " Problèmes régionaux " (France, Europe centrale et orientale, exemples méditerranéens, scandinaves et cas particuliers de la Pologne et de la Roumanie).

C'est ainsi qu'A. Soboul ouvre la première partie avec l'étude de la " féodalité tardive " (p. 35-49). On retrouve ici l'essentiel des réflexions de Soboul sur les problèmes du prélèvement féodal-seigneurial et de la " transition ". Retenons surtout qu'il n'y a pas un mais des systèmes et que de nombreuses variables s'observent à l'intérieur même d'un système donné : le " cas français " l'illustre fort bien et de récentes études ont précisé ces variations de « pays " à " pays", voire d'une seigneurie à l'autre?.

Fondements sociaux, ensuite, avec la noblesse (J. Meyer), la bourgeoisie (É.H. Balázs et E. Niederhauser), les " intellectuels " (D. Kosáry) et la paysannerie (I. Wellmann). Et l'on retrouve, par ces contributions, la question essentielle de la « nature sociale de l'État absolutiste " : État d'équilibre entre noblesse et bourgeoisie ou prééminence de l'aristocratie? L'article d'É. Balázs et $\mathbf{E}$. Niederhauser suggère certaines réponses auxquelles il nous semble possible de souscrire. La centralisation et la laïcisation qui caractérisent la plupart des régimes d' " absolutisme éclairé " conduisent au développement d'un " corps de fonctionnaires spécialisés d'origine roturière " (p. 67) et les auteurs reprennent à leur compte cette hypothèse : "l'absolutisme éclairé a plutôt engendré une future bourgeoisie moderne qu'il n'a coopéré avec une bourgeoisie trouvée sur place " (p. 67). Constatation, somme toute, assez voisine de la formule de Daniel Dessert : « la bourgeoisie comme entité socio-économique est une fille involontaire de l'État d'Ancien Régime et de sa société aristocratique ${ }^{8}$.

Quelle est alors l'originalité française? C'est, bien sûr, la Révolution. Se

5. Il s'agit de la reproduction de la conclusion de l'ouvrage de Jean MeYer, Noblesse et pouvoirs dans l'Europe d'Ancien Régime, Paris, Hachette, 1973.

6. Perry Anderson, L'État absolutiste. I : L'Europe de l'Ouest, Paris, Maspero, 1978, p. 18. L'ouvrage est mentionné dans sa version originale en langue anglaise.

7. Entre autres les travaux de Jean BASTIER sur le Toulousain (1975), de Philippe GouJARD sur le pays de Bray (1979), de Jean-Noël Luc sur la Charente-Inférieure (1984).

8. Daniel Dessert, Argent, pouvoir et société au grand siècle, Paris, Fayard, 1984, p. 429. 
pose ici le problème des relations entre les «intellectuels » et le (ou les) pouvoir(s). Objet de la réflexion de $\mathrm{D}$. Kosáry à partir de l'exemple hongrois (p. 71-79), cette interrogation s'inscrit aussi au centre de la contribution de B. Köpeczi (« L'idéologie de l'absolutisme éclairé », p. 101-118), se retrouve dans le travail de G. Ricuperati ("Eglises et Lumières ", p. 119-132) et dans celui de W. Markov ( Princes et administrateurs éclairés : l'individu et l'histoire ", p. 143-157 - en allemand, sauf la conclusion). Enfin, parce que la réforme du système éducatif fut au cœur des préoccupations des monarques (ou des ministres) éclairés, la question du statut et du rôle des intellectuels figure dans la plupart des contributions de la seconde partie, consacrée aux problèmes régionaux. L'Europe intellectuelle avait eu les yeux tournés vers l'Angleterre, mais aussi vers Berlin, Pétersbourg, Parme, Vienne, Lisbonne, Florence, Copenhague... À partir de 1789, l'attention se focalise sur la France et sa révolution, " préparée " par les philosophes et largement conduite, " en haut ", par des intellectuels (il n'est qu'à regarder la composition des Assemblées révolutionnaires pour s'en convaincre). “ On assista ainsi, dit G. Ricuperati, à une lente évolution des intellectuels réformateurs [...] Ou l'on se transformait en Jacobin, ou l'on rentrait dans les rangs de la tradition " (p. 131). Si nous ne partageons pas l'opposition induite par G. Ricuperati entre "Lumières " et " Jacobinisme " (ibid.), nous nous trouvons en accord avec la chronologie suggérée : c'est dès son déclenchement que la Révolution française suscita, en Europe, un clivage entre partisans et adversaires. Les " excès de la Terreur ", s'ils furent immédiatement essentiels dans les lignes de partage politiques en France même puis, au XIXe siècle, en Europe, n'eurent sur le moment, qu'une faible incidence à l'extérieur. Kant pouvait ainsi écrire, en 1798 : «Que la révolution d'un peuple spirituel que nous avons vu s'effectuer de nos jours réussisse ou échoue; qu'elle amoncelle la misère et les crimes affreux [...] - cette révolution, dis-je, trouve néanmoins dans les esprits de tous les spectateurs [...] une sympathie d'aspiration qui touche de près à l'enthousiasme " (Le Conflit des Facultés, 2e section, § 6).

Plus déterminants furent sans doute la guerre, l'appétit conquérant de la Grande Nation et l'arrivée au pouvoir de celui que Fichte appelait « l'homme sans nom ". Le règne de Napoléon, concluent É. Balázs et E. Niederhauser, ne peut en aucun cas être considéré comme une expérience d'absolutisme éclairé (thèse admise, en revanche, par A. Soboul, p. 25), pas plus que l'absolutisme éclairé ne survit en Europe de l'Est aux guerres napoléoniennes (p. 68).

Les contributions régionales tendent à nuancer cette datation terminale. Si tous les auteurs attribuent un grand rôle aux événements français de 1789-1815, ils se séparent nettement sur l'importance à accorder aux facteurs endogènes ou exogènes dans l'échec de l'absolutisme éclairé. F. Diaz écrit ainsi du projet de constitution de Pierre-Léopold (élaboré entre 1778 et 1782) qu'il « représentait à la fois le point le plus élevé de l'absolutisme éclairé en Italie et son arrêt de mort. [...] Le prince Habsbourg, pris entre ses doutes sur la maturité civile des Toscans, les difficultés intérieures et celles que lui créaient ses rapports avec son frère empereur, les incertitudes et les anxiétés suscitées par le début de la Révolution française, finit par ne pas appliquer sa constitu- 
tion » (p. 269). Appréciation que l'on retrouve dans le cas espagnol étudié par M. Kossok : "Bien que personne ne mette en doute que la Révolution de 1789 a causé une rupture dans l'évolution historique, le problème de savoir quels étaient les facteurs intérieurs [...] n'est pas du tout résolu " (p. 288) et l'auteur souligne les divergences au sein du parti réformiste (démission d'Aranda) avant la mort de Charles III en 1788, les résistances de l'aristocratie aux mesures agraires de 1770 et la crise de la domination coloniale. Pour G. Klingenstein, l'échec du "joséphisme " date, non de 1790-1792, mais du règne même de Joseph II : opposition des masses rurales à l'Édit de Tolérance de 1781 , révolte des paysans roumains contre les seigneurs hongrois de Transylvanie en 1785 qui conduit l'aristocratie à refuser la Patente sur les terres. « Sous les nouvelles charges de la guerre contre les Turcs (1788-1789), conclut l'auteur, les peuples - noblesse, bourgeoisie et paysannerie - refusèrent d'accorder leur confiance à Joseph. L'exemple de la France avait un effet stimulant " (p. 215).

Sans multiplier les exemples, retenons deux cas extrêmes. Pour les États allemands, E. Weiss insiste sur la continuité entre les réformes de l'absolutisme éclairé et celles introduites entre 1803-1806 et 1821 (p. 198-199). Au Portugal, en revanche, " la mort du roi José Ier et la chute de Pombal (1777) ont marqué la fin de l'absolutisme ministériel éclairé » (H. Bach, p. 297).

Restent enfin le contenu de l'absolutisme éclairé et le problème du " modèle " prussien, étudié par Ingrid Mittenzwei (p. 171-187). Sans reprendre les débats historiographiques sur le "roi-philosophe ", on peut s'interroger sur la représentativité et/ou l'originalité de l'œuvre frédéricienne. Appartiennent aux traits généraux de l'absolutisme éclairé la réforme judiciaire laborieusement achevée en 1782, la tentative (même avortée) d'abolition du servage en Poméranie, la relative liberté accordée à la presse et aux écrivains. Mais la spécificité politique de Frédéric II réside sans doute dans son ambition pour imposer la Prusse comme "grande puissance" dans le jeu de l' "équilibre européen " (la notion triomphe au XvIII siècle). Pour y parvenir, Frédéric usa de deux moyens, la guerre et le développement économique. Il s'attacha ainsi à faire naître une conscience politique prussienne et encouragea les créations industrielles, essentiellement dans les provinces moyennes (Marche électorale, par exemple). Il combina un protectionnisme rigide et l'appel, accru après 1763, à des spécialistes et des techniciens étrangers (sans oublier l' "espionnage industriel » aux dépens de l'Angleterre). Dira-t-on que ce n'était, après tout, qu'une forme de "colbertisme" ? Rien n'est moins sûr et il semble impossible, à se tourner vers le xıx ${ }^{\mathrm{e}}$ siècle - au-delà de "l'effondrement d'Iéna " (A. Soboul, p. 24) - de nier l'originalité prussienne ; les sujets des Hohenzollern n'avaient-ils pas intériorisé cette constatation de l'Anti-Machiavel: «Quant aux manufactures de toutes espèces, c'est peut-être ce qu'il y a de plus utile et de plus profitable à un État, puisque, par elles, on suffit aux besoins et au luxe des habitants, et que les voisins sont même obligés de payer tribut à votre industrie ; elles empêchent, d'un côté, que l'argent sorte du pays, et elles en font rentrer de l'autre " (chap. xxI)?

Au terme de ce compte rendu, c'est surtout la diversité des études qui nous 
semble faire la richesse de cet ouvrage. Diversité des contributions : les points de vue exprimés sont, en effet, pluriels et l'on doit rendre hommage à cette volonté, affirmée par les responsables de la publication, de donner au livre un " caractère multicolore ". Diversité, aussi, des situations régionales. À tel point que, sans douter de la tragique originalité de la Pologne étudiée par Z. Libiszowska ( «il ne s'agissait plus de rationaliser l'État, mais de le sauver ", conclut l'auteur, p. 329), ou des particularités de la Moldavie et de la Valachie (A. Dutu, p. 331-337), on peut se demander si l' «absolutisme éclairé " a bien existé ou s'il ne conviendrait pas de parler d' " absolutismes éclairés ». Ce n'est pas le moindre mérite de ce volume que de faire prendre conscience du danger des généralisations hâtives. Enfin, l'importante bibliographie établie par E. Tamási «d'après les recommandations des auteurs " (p. 339-361) constitue, en dépit de certaines lacunes (qui tiennent aussi au retard de la publication), un guide fort précieux. En conclusion, un instrument de travail de référence.

Françoise BrunEL.

Robert Darnton, Le Grand massacre des chats. Attitudes et croyances dans l'ancienne France. Trad. de l'américain par Marie-Alyx Revellat. Paris, Laffont, 1985. $15 \times 24,282$ p., index ("Les hommes et l'histoire").

Les problèmes posés par ce livre de Robert Darnton sont assez bien résumés par ce titre trompeur muni d'un sous-titre explicatif. Les chats, en effet, ne miaulent que durant une vingtaine de pages (p. 75 à 99) et cèdent la place à d'autres sujets, mais l'association de l'animal familier-mystérieux et de la violence débridée est apte à attirer le lecteur. Et c'est bien ainsi, car ce livre est destiné au grand public qui a ici l'occasion de pénétrer, de plaisante façon, dans les mentalités du xvıII siècle. Titre et sous-titre illustrent le rapport entre le fait divers exemplaire car surprenant et le débouché qu'il permet sur un monde d'attitudes et de croyances oubliées. Dans cette articulation s'inscrit la conviction de R. Darnton : les mentalités du passé sont pénétrables dans leurs surgissements les plus opaques. La matière de l'historien des mentalités est d'abord étonnement.

L'introduction nous invite au voyage dépaysant qui nous conduit dans les cultures populaires, bourgeoise, littéraire. Première étape : le sens des contes des veillées d'antan. L'auteur écarte l'explication psychanalytique (Fromm, Bettelheim) en montrant qu'elle retient des éléments narratifs que la genèse des textes fait apparaître comme accessoires et tardifs. Le psychanalyste pèche par anachronisme, il " nous entraîne dans un univers mental qui n'a jamais existé, en tout cas pas avant la naissance de la psychanalyse » (p. 17). Les contes ne prennent leur sens véritable que lorsqu'ils sont mis de plain-pied avec les réalités d'Ancien Régime, c'est-à-dire quand ils apparaissent comme des guides pour affronter un monde dominé par la violence et la ruse. La leçon est magistrale, elle montre combien, sans compréhension historique, la 
recherche du sens tourne le dos aux réalités humaines. La relativité due à l'épaisseur historique, au contraire, montre tout ce que ces dernières ont de têtu face aux schémas du prêt-à-interpréter.

C'est à une mise en perspective de même type que se livre ensuite l'auteur pour expliquer un massacre de chats par des compagnons imprimeurs. $\mathrm{Ce}$ massacre évidemment empli de symboles s'inscrit à la confluence des pratiques «folkloriques " et des mours et conditions sociales de l'imprimerie du Xviri ${ }^{\mathrm{e}}$ siècle (si bien connue de R. Darnton). Sont ensuite étudiées les perceptions de sa ville par un bourgeois de Montpellier qui nous livre sa vision des classes et des ordres - et de l'ordre - urbains, du monde des " intellectuels " par un inspecteur du commerce du livre, de la classification des connaissances par l'Encyclopédie, de la lecture par Rousseau et ses admirateurs.

R. Darnton met ainsi en évidence des problèmes centraux pour l'historien des mentalités : sens de la classification, sens du symbole, sens d'un acte intellectuel. L'analyse repose sur une conviction, l'altérité du passé, un but, la compréhension de l'humain, une méthode, la mise en perspective culturelle d'un fait isolé révélé par des archives originales.

Dans son introduction et surtout dans sa conclusion, l'auteur est pleinement conscient de la précarité de sa tâche. Attardons-nous, avec lui, sur ce point. Si l'anecdote est une clef pour pénétrer le passé, ne comporte-t-elle pas un danger réductionniste? Le choix de l'histoire qualitative, au détriment d'un dépouillement quantitatif du troisième niveau (c'est-à-dire du culturel) séduit l'esprit mais la séduction n'implique pas la conviction d'une saisie totale. N'y a-t-il pas un a priori à proclamer l'altérité totale des mentalités passées ? Si le temps est une frontière, il est, comme toute frontière, perméable et je ne suis pas persuadé que la lecture rousseauiste, pleine de passion dévorante, ait, par exemple, perdu tout adepte. En terminant sur une citation de Marc Bloch (« le bon historien ressemble à l'ogre de la légende. Là où il flaire la chair humaine, il sait que là est son gibier "), R. Darnton achève sa profession de foi impressionniste. Encore faudrait-il préciser que la chair de cet ogre et celle de son gibier sont consanguines : l'historien est le produit final de cette culture dont il dégage et analyse les strates. En ce sens, le rapport à son objet d'étude me semble très différent de celui de l'anthropologue sur lequel $R$. Darnton s'est appuyé tout au long de ce livre pour justifier sa démarche : l'historien de la culture est trop nécessairement anthropophage pour être pleinement anthropologue.

Si cette promenade dans le passé est suggestive, son balisage théorique reste hésitant. Sur cette hésitation, les historiens de la culture et des mentalités ont encore beaucoup à réfléchir.

Pierre Monzani. 
Louis PérouAs, Refus d'une religion. Religion d'un refus en Limousin rural, 1880-1940. Paris, E.H.E.S.S., 1985. $15 \times 22,5,245$ p., bibliogr., cartes, index.

Le P. Louis Pérouas s'est fait connaître, voici une vingtaine d'années, en inaugurant avec son Diocèse de La Rochelle de 1648 à 1728 (Paris, 1964) les travaux d'une riche école de sociologie et d'anthropologie religieuses de l'époque d'Ancien Régime, illustrée aussi par A. Dupront, J. Delumeau, M. Vovelle, B. Plongeron, puis leurs élèves. Au cœur de sa problématique, suscitée par les interrogations de G. Le Bras et F. Boulard sur l'état de la pratique et du sentiment religieux en France vers le milieu du $x^{e}$ siècle, L. Pérouas inscrivait la recherche des origines du profond dimorphisme opposant le très fervent bocage vendéen et l'indifférence des plaines qui lui succèdent au sud. Cette mission accomplie, l'auteur a choisi de s'établir plus à l'est, dans ce Limousin dont l'état de " déchristianisation » - terme à manier avec précaution, rappelle-t-il, après d'autres - fascinait déjà Gabriel Le Bras dès les années 1930, spécialement le département de la Creuse, devenu l'archétype du « pays de mission ».

Par rapport au diocèse de La Rochelle, tant la méthode que les résultats ont sensiblement évolué. D'abord, parce que le choix a été fait pour une méthode explicitement régressive, partant du point d'aboutissement, pas contemporain mais presque, en gros la période de la Troisième République, un ouvrage ultérieur devant décrire une fouille remontant jusqu'au Xve siècle. On pense ici à Paul Bois remontant lui aussi les siècles dans la Sarthe. Seulement le dimorphisme interne au champ religieux, et qui occupe tout le livre, n'est pas celui décrit dans tant d'autres études de sociologie électorale ou religieuse, où le chercheur se plaît à suivre une frontière entre des territoires contrastés, et à aligner tous les écarts corrélatifs possibles. Car le Limousin est massivement tiède, voire indifférent, excepté en ses marges auvergnates, la Combrailles ou la Xaintrie. Il illustre le refus de la religion catholique, ici suivi pas à pas dans la fidélité aux catégories lebrasiennes : d'abord, le détachement de la pratique pascale, jadis pierre de touche d'un comportement religieux « régulier », puis abandon, plus original en France, des grands rites de passage et de la pratique « saisonnière " elle-même, baptêmes, mariages, obsèques. L'ensemble fait tache d'huile à partir de la Creuse, puis déborde sur les départements voisins par la Montagne limousine, sur la Haute-Vienne, puis sur la Corrèze. Le mouvement s'est vivement accéléré après la Séparation de 1905, mais était déjà bien amorcé. La recherche des corrélations, tant intrareligieuses (recrutement, qualité et action du clergé local) qu'extrareligieuses (industrialisation à partir de Limoges, rôle des migrations dans ce château d'eau démographique) n'autorisent pas d'explication univoque. Ainsi l'émigration, bête noire du clergé de la fin du $x^{e} x^{e}$ siècle en Limousin comme ailleurs, peut mener, ici, au détachement profond, là, ne pas empêcher la fidélité.

Force est donc de ne pas en rester au seul refus de la religion en Limousin rural, mais de bien voir que ce refus a, en soi, une dimension religieuse. L'apport le plus neuf du travail de L. Pérouas est, en effet, pour la première 
fois à ce point de microanalyse, l'étude très fine de ce que d'autres ont appelé, sur un plan très général, religions de remplacement, ou religions "séculières " (Raymond Aron, etc.). Il existe, en effet, moins une indifférence religieuse, au sens que lui donnaient Lamennais ou F. Boulard, qu'une « foi dans un idéal areligieux ". Un authentique rationalisme populaire - l'évangélisme protestant se brise ici les dents après le catholicisme tridentin - se cherche à travers des liturgies de substitution, mariages, baptêmes et surtout sépultures civiles, qui finissent d'ailleurs par produire les mêmes effets de conformisme, à l'envers, que dans les régions de catholicisme grégaire. Au passage, l'auteur nous livre de précieuses indications méthodologiques, transposables en d'autres lieux, sur le calcul de la proportion de sépultures civiles.

Deux questions restent posées, une fois le livre refermé, en regardant vers l'amont et vers l'aval, vers le passé et vers l'avenir. Pourquoi ce détachement, de plus en plus militant, du catholicisme, dans cette région précisément, ce bocage qui ressemble finalement beaucoup à ceux de l'ouest ? Autrement dit, pourquoi la Réforme catholique du Xvile siècle, et le Réveil du xixe siècle ultramontain, ont-ils échoué ici, pas ailleurs, permettant le succès des religions de remplacement, jusqu'au communisme, d'ailleurs peut-être un peu négligé dans l'ouvrage. Alain Corbin nous a dit naguère ce qu'il en pensait, dans son Archaisme et modernité en Limousin, 1845-1880 (Paris, Marcel Rivière, 1975) et nous attendons avec impatience le second volume, déjà entrevu dans quelques articles, promis par L. Pérouas. La deuxième question porte sur la comparaison de ce détachement, très daté, situé et typé, avec celui qui s'opère aujourd'hui sous nos yeux, toutes régions confondues, et qui gagne en particulier les couches jeunes de la population. N'aurait-on pas ici un phénomène radicalement nouveau, puisque les religions du refus, politique et idéologique, ont elles aussi à en pâtir? Comme on aimerait savoir, mais il y faudrait un troisième livre, ce qui se passe en Limousin aujourd'hui même, près d'un demi-siècle après la borne choisie par Louis Pérouas.

Michel LagréE.

Le Personnel de l'enseignement supérieur en France aux XIX et Xx siècles. Éd. par Christophe Charle et Régine Ferré. Colloque organisé par l'Institut d'histoire moderne et contemporaine et l'École des hautes études en sciences sociales les 25 et 26 juin 1984. Paris, C.N.R.S., 1985. $16 \times 24$, 284 p., index.

La publication des actes de ce Colloque marque une étape décisive à l'intérieur de deux parcours de recherches dont il a eu, avant tout, le mérite de réaliser la rencontre : celui de l'histoire de l'enseignement supérieur qui représente, dans divers contextes nationaux, un domaine classique et connaît un progrès dans l'historiographie contemporaine ; celui de la prosopographie des élites en France, un sujet de recherche «lancé » en 1979, justement par l'Institut d'his- 
toire moderne et contemporaine à l'École normale supérieure, alors dirigé par Jean-Marie Mayeur, auteur de la préface de ce volume.

Après six ans, cette recherche s'est révélée un secteur d'exploration très fécond et innovateur en particulier en ce qui concerne l'étude des élites dans la la haute fonction publique. Il n'est pas surprenant que le secteur de l'enseignement supérieur ait représenté, à l'intérieur de cette recherche, un territoire pourvu d'une forte cohérence thématique. Dans le Guide de recherche qui traçait le programme de cette étude, on révélait, en effet, que « les universitaires rassemblent les conditions apparemment optimales pour l'étude d'une élite : définition simple, informations et sources abondantes, corps attaché à entretenir la mémoire des défunts ". En même temps, on soulignait le caractère désuet, pour ne pas dire archaïque, de l'interprétation historiographique et d'un corps bibliographique riche, mais construit à partir de perspectives conventionnelles.

Les apports innovateurs de ce volume semblent donc "surdéterminés". D'une part - surtout dans la première section "Sociologie du corps enseignant $»-$, en intégrant des analyses des institutions et de la dynamique des élites, il a réalisé un nouveau type de polarisation des études sur l'enseignement supérieur, non plus seulement centrées sur des processus d'institutionnalisation disciplinaire, mais sur les agents sociaux qui ont réalisé la formation des corps universitaires (les maîtres de conférences, dans l'article de Françoise Mayeur; les professeurs du Collège de France, dans celui d'A. Lechat; les professeurs de l'Académie de médecine, dans la recherche de G. Weisz). D'autre part, en utilisant également la riche contribution offerte dans ce secteur par les travaux des chercheurs anglo-saxons, ce volume assume un rôle de premier plan, pour la dissolution de ce que l'on peut considérer comme le principal idolum tribus dans ce domaine de la recherche, du moins en ce qui concerne la France : le perspicace attachement de l'historiographie traditionnelle à la constatation que le système universitaire national était très en retard, car il privilégiait le moment pédagogique-éducatif dans les fonctions de l'enseignement, et de ce fait aurait freiné le développement de la recherche.

Cette deuxième orientation constitue le principal fil conducteur qui traverse les trois sections du volume, même s'il n'assume sa pleine identité thématique que dans la troisième partie : "Contribution de l'enseignement supérieur à la recherche et au changement culturel."

Dès la première section, l'article de V. Karady, "Lettres et sciences. Effets de structure dans la sélection et la carrière des professeurs de faculté (1810-1914) », est entièrement centré sur les trajectoires qui modifient, au cours de la période considérée, les rapports entre l'Université et le secteur intellectuel extra muros, à l'avantage de la première. Si, dans la première moitié du XIX $x^{e}$ siècle, le milieu intellectuel est traversé par des mouvements scientifiques importants qui expriment, en même temps, un défi au monde universitaire, "sous la forme d'une demande sociale que les facultés peuvent de plus en plus mal satisfaire ", et un rappel au retard de l'Université française par rapport aux réalisations scientifiques des Universités étrangères, la situation change radicalement au cours de l'heureuse conjoncture des décennies quatre- 
vingt/quatre-vingt-dix. Grâce à l'introduction « de la performance scientifique comme critère essentiel de sélection dans le supérieur..., l'universitaire se définit désormais dans les facultés par l'activité scientifique plus que par l'enseignement qu'il dispense ". La recherche devient donc un trait distinctif de la communauté scientifique non seulement grâce à la création d'institutions parallèles à l'enseignement universitaire comme l'École pratique des hautes études, mais également grâce aux Facultés elles-mêmes. C'est à travers la recherche que le monde universitaire impose ses propres critères de « distinction " - et de force - par rapport aux savants " amateurs". À la fin du siècle, les universitaires contrôlent les principaux instruments de la naissance du marché scientifique (direction de revues, comités scientifiques, instituts de recherche). " La marginalité - dans ce marché -, conclut Karady, se mesure dorénavant par la distance à l'institution universitaire. "

$\mathrm{Si}$, pour les Facultés scientifiques - et pour les provinciales en particulier, comme le démontre l'article de R. Fox, « l'attitude des professeurs des Facultés des sciences face à l'industrialisation en France entre 1850 et 1914 " -, cette rupture semble élargir effectivement le pouvoir des Facultés de répondre, à travers la recherche appliquée, à la croissante demande sociale du monde des affaires et de l'industrie, le discours paraît beaucoup plus complexe et hérissé de difficultés méthodologiques en ce qui concerne le secteur des sciences humaines.

En outre, il faut également rappeler que, pour le secteur scientifique, Robert Fox lui-même a mis en évidence, malgré des éléments convergents révélés par les recherches récentes sur " la dévotion des professeurs à l'utilité économique ", d'autres circonstances qui se sont opposées à une réelle coordination des activités de l'Université et du monde de la production et qui induisent, au moins, à la prudence lorsque l'on expose cette thèse. "Virtuellement, écrit Fox, tous les professeurs pouvaient, et beaucoup le faisaient, déclarer publiquement qu'ils désiraient servir l'économie. Mais il était trop tentant, en pratique, de chercher à servir l'économie en changeant le moins possible les cycles d'études et les habitudes. " Ces observations sont " surdéterminées " si on les applique aux Facultés humanistes, dans lesquelles, jusqu'au début des années cinquante, il manque une réelle incitation à la "professionnalisation " des sciences humaines en termes de recherche appliquée. Donc, si le tracé de la problématique de Victor Karady, qui - comme on l'a vu - a efficacement montré comment, à la fin du siècle dernier, le modèle scientifique des disciplines littéraires, fixé par les professeurs universitaires, s'est révélé stimulant et innovateur, son extension aveugle peut devenir paralysante. À ce propos, je voudrais citer un article récent de $\mathrm{O}$. Dumoulin, publié dans la Revue française de sociologie (vol. XXVI, 21, 1985), qui analyse, justement à partir de ce tracé problématique, la préhistoire du C.N.R.S., pour le secteur des sciences humaines. Probablement fourvoyé par une documentation incomplète l'auteur déclare, en effet, qu'il n'a pas consulté les archives du C.N.R.S. - , Dumoulin accuse la création de la Caisse nationale de la recherche et surtout du Conseil supérieur de la recherche scientifique, d'avoir consommé définitivement la « rupture épistémologique » entre " amateurisme " érudit et litté- 
raire et recherche scientifique, en soutenant que la Caisse nationale de la recherche opposait nettement "recherche nationale " et érudition locale. Un examen attentif des procès-verbaux de la IVesection, celle des sciences humaines, se trouvant justement dans les archives du C.N.R.S., lui aurait permis d'observer que les sociétés savantes étaient autant, sinon majoritairement, " représentées » que la recherche universitaire et que - selon «l'esprit du temps " - le Conseil supérieur de la recherche scientifique fonctionnait surtout comme " organisme de représentation des intérêts de la recherche ", plus orienté vers " l'organisation des intérêts " que vers la " professionnalisation" à laquelle l'auteur fait référence explicitement.

Sur ce terrain, il a été probablement fourvoyé par son attirance pour une problématique qui est en train de devenir à la mode, mais qui, pour être « intériorisée " dans le contexte européen, aurait besoin de plus grandes recherches que celles qui nous sont offertes par l'auteur de cet article. Et cela surtout pour la période précédant la Deuxième Guerre mondiale, durant laquelle la problématique de la "professionnalisation " semble difficilement applicable au système de l'enseignement supérieur européen, inséparable du contexte, non seulement théorique-historiographique, mais également socio-historique où elle apparaît : celui de l'organisation du système universitaire américain, pendant l'époque progressiste et de sa particulière interpénétration avec la croissance du " corporate capitalism ", grâce à l'institutionnalisation d'un réseau articulé d'associations professionnelles, fonctionnant comme des "agences" de recherche reliées à la société et au monde industriel, et qui est presque inexistant dans le contexte européen. Dans ce sens, en ce qui concerne la France, il est difficile de parler des processus de légitimation scientifique de la recherche, à travers lesquels s'affirme le monopole universitaire des sciences humaines par rapport à l'amateurisme de l'érudition, comme d'une « étape de leur professionnalisation ".

Sous ce profil, je crois qu'il faut considérer comme très appréciable la contribution offerte au Colloque par A. Drouard, « À propos du statut de la sociologie en France ". En effet, l'auteur souligne les obstacles opposés, non seulement par l'enseignement universitaire mais également par l'organisation de la recherche, à la professionnalisation de la sociologie, avant la fin des années soixante : "Avant la création de la licence en sociologie, écrit Drouard, [...] l'enseignement reste tout aussi rhétorique, spéculatif et magistral, même si la mise en place des travaux dirigés s'inscrit dans la perspective d'une formation méthodologique. De même, le doctorat de Troisième cycle est créé sans moyens pour le faire réussir et sans initiation à la pratique de la recherche. " Lucette Le Van-Lemesle a, pour sa part, une attitude plus défensive à propos du rôle des professeurs de la Faculté de droit dans la " professionnalisation " de l'économie politique, au cours des années qui précédèrent la Deuxième Guerre mondiale. En soulignant l'engagement des professeurs d'économie politique pour la recherche et ses applications, l'auteur relève qu'il s'est inséré dans un contexte social et politique "qui [voulait] préserver ses structures, "l'équilibre " entre l'agriculture, l'industrie et les services, la " complémentarité ", entre les petites, moyennes et grandes entreprises ... [dans] une 
société qui ne souhait[ait] pas une diffusion massive de ce qui pou[v]ait menacer sa reproduction ». L. Le Van-Lemesle semble ainsi négliger le rôle joué par certaines fractions spécifiques " d'intellectuels de l'industrie ", ingénieurs et hauts fonctionnaires des services publics, dans la transformation de la fonction de cette discipline en véritable instrument de policy-making, justement durant les années qui précédèrent la Deuxième Guerre mondiale.

Toutefois, il est indiscutable que l'articulé et intelligent programme du Colloque a permis de neutraliser, avant même qu'il puisse prendre corps, le danger, lié à tout parcours réellement et sciemment innovateur, qui serait ici de remplacer l'idée d'un retard généralisé de l'enseignement supérieur français, face aux modèles étrangers, par celui de sa modernité à tout prix.

Giuliana Gemell.

\section{ÉTUDES SUR SOREL}

Cahiers Georges Sorel, 3, 1985.

Le numéro 3 des Cahiers Georges Sorel confirme les buts de la jeune Société d'études soréliennes, née des suites du Colloque tenu à l'École normale supérieure de la rue d'Ulm au printemps 1982 . En effet, si le colloque se présentait comme un état des études soréliennes dans le monde, la création de la Société correspondait au souhait de développer autour de la personne et de l'œuvre de Georges Sorel de nouvelles recherches. Il ne s'agissait ni de se livrer à une pure exégèse de l'œuvre de Sorel (chez qui, pourtant, les apparentes contradictions théoriques et la disparité des moyens d'expression justifient sans doute plus que chez beaucoup d'autres une lecture verticale), ni de tomber dans le travers d'une amicale sorélienne en publiant " les notes de blanchisseuses ", ni d'ouvrir la voie à d'éventuelles extrapolations d'ordre politique. La Société se donnait pour tâche de publier Sorel, dans le but de contribuer à une meilleure connaissance du monde intellectuel, socialiste et ouvrier du tournant du siècle.

L'cuvre du "solitaire de Boulogne " mérite attention à plusieurs titres : parce que s'y lisent les grands débats de la fin du $x_{1} x^{e}$ et du début du $x^{e}$ siècle, du syndicalisme révolutionnaire au fascisme, parce qu'elle est celle d'un ingénieur dont le destin personnel n'est pas indifférent, parce qu'elle est paradoxale et ambiguë. Peu lu ou mal lu, Sorel est porteur de lourdes postérités en mal de pères spirituels. La tentative des Cahiers est bien de fonder autour d'un penseur oublié ou masqué par ses héritiers une revue d'histoire intellectuelle, qui ne soit pas une pure histoire des idées. Et Sorel, au cœur d'un réseau européen de correspondants, du philosophe hégélien Croce au sociologue Roberto Michels, du socialiste "révisionniste " Bernstein à l'économiste Vilfredo 